\title{
Toxicity, Pharmacokinetics, And Antitumor Activity Study of a Novel Long- Acting Paclitaxel Nanodrug Delivery System Constructed On a Curcumin Derivative Carrier
}

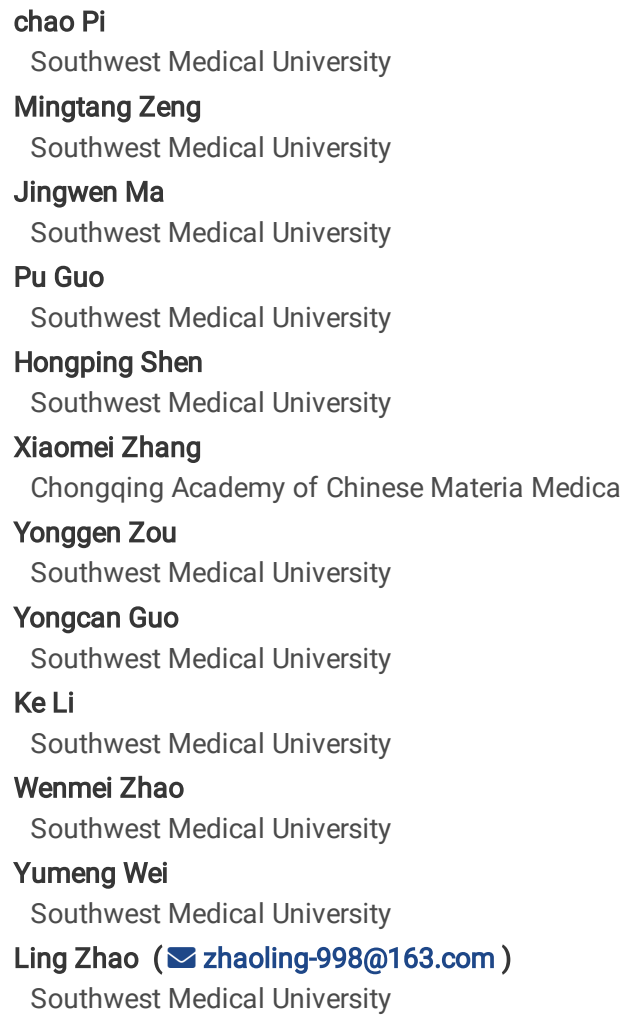

\section{Research Article}

Keywords: breast cancer, paclitaxel, curcumin derivatives, nanoparticles

Posted Date: September 20th, 2021

DOI: https://doi.org/10.21203/rs.3.rs-877478/v1

License: (9) (7) This work is licensed under a Creative Commons Attribution 4.0 International License. Read Full License 


\section{Abstract}

Purpose/Background: Paclitaxel (PTX) has been widely used in the clinic to treat breast cancer. However, its poor water solubility and intolerable toxicity greatly reduce the efficacy and medication safety. In this paper, a curcumin derivative (CUD) synthesized by the research group was used as a carrier to prepare a new type of PTX nanodrug delivery system (CUD-PTX-LN), thereby improving the safety of PTX medication and enhancing the anti-tumor effect.

Methods: CUD-PTX-LN was prepared by solid dispersion technology and the dialysis method was adopted to investigate the release behavior of CUD-PTX-LN in vitro. Meanwhile, the dynamic process of nanoparticles in vivo was analyzed by pharmacokinetic experiments. Hemolysis experiment, acute toxicity and cumulative toxicity were conducted in mice to evaluate the safety of CUD-PTX-LN. Subsequently, the anti-tumor activity of CUD-PTX-LN was investigated using in vitro and in vivo breast cancer cell models.

Results: The average particle size, PDI, and Zeta potential of CUD-PTX-LN were $238.5 \pm 4.79 \mathrm{~nm}, 0.225 \pm 0.011$ and $-33.8 \pm 1.26 \mathrm{mV}$, respectively. CUD-PTX-LN had an encapsulation efficiency (\%EE) of $94.20 \pm 0.49 \%$ and an achievable drug loading (\%DL) of $10.98 \pm 0.31 \%$. This nanoparticle was stable for up to half a year when the lyophilized powder was stored at room temperature. Cytotoxicity test on MCF-7 cells demonstrated the stronger cytotoxic activity of nanoparticles in comparison to free PTX. CUD-PTX-LN significantly prolonged the half-life and retention time of PTX. The area under the curve (AUC) of CUDPTX-LN was $14.72 \mathrm{mg} / \mathrm{L} \times \mathrm{h}$, which was 6 times that of the free PTX group ( $2.38 \mathrm{mg} / \mathrm{L} \times \mathrm{h}$ ). In anti-tumor experiments in vivo, CUD-PTX-LN possessed the advantages of improving the antitumor efficacy and reducing the toxic side effects of drugs. Importantly, CUD-PTX-LN is safer than free PTX in terms of assessing hemolytic, acute and cumulative toxicity.

Conclusion: A novel PTX nanodrug delivery system constructed based on CUD as a carrier holds great potential in improving breast cancer treatment.

\section{Introduction}

Breast cancer, a malignant tumor with a predominantly female patient population, has become a major disease that seriously threatens women's health [1]. Globally, the incidence and mortality of breast cancer have gained momentum in recent years, resulting in a more severe disease burden on society [2]. In view of this, there is an imminent need to find and develop safer and more effective therapeutic agents for breast cancer.

Paclitaxel (PTX) as a first-line chemotherapeutic agent has exhibited obvious therapeutic advantages in the treatment of breast cancer, ovarian cancer, cervical cancer and lung cancer [3-6]. However, as with other chemotherapeutic agents, PTX-related preparations often cause intolerable toxicity in patients, although they bring prominent therapeutic effects for clinical antitumor therapy. Hemolysis, myelosuppression and hypersensitivity are typical serious adverse reactions of PTX [7-11]. These toxicities not only reduce the patient's treatment compliance, thereby limiting the clinical application of PTX, even threatening the safety of patients' medication. In addition, defects such as poor aqueous solubility and rapid blood clearance also hinder the development of PTX [12]. In order to better exert the anti-tumor value of PTX, technological innovations are urgently needed to further improve its therapeutic efficacy and safety.

Nanotechnology has shown great potential to address the frequently occurring problems in conventional chemotherapy of PTX by altering the pharmacokinetic and release behaviors of drugs, thereby improving therapeutic efficacy and reducing systemic toxicity [13]. Among them, various nanodrug delivery systems based on carriers have been widely investigated for tumor applications, protecting drugs from rapid clearance in the blood, and preferentially accumulating in solid tumors via the enhanced permeability and retention (EPR) effect [14]. The passive accumulation of carrier materials at the tumor site mediated by the EPR effect can improve the bioavailability of drugs and alleviate the toxic side effects of systemic chemotherapy [15]. Some carrier based PTX nanodrug delivery systems were experimentally proven to be effective as well $[16,17]$. However, part of the carriers: $₫$ low drug loading capacity, $₫$ high

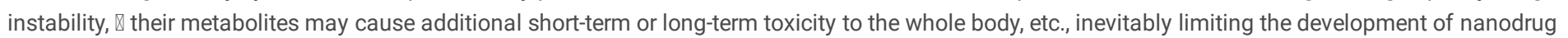
delivery systems based on carrier materials $[18,19]$. Therefore, the search for carrier materials with simple preparation methods, high therapeutic efficacy, low side effects and great biocompatibility is the main goal of current research in nanodrug delivery systems.

Curcumin (CU), a natural Dione polyphenolic substance, can exert broad-spectrum anticancer effects through a variety of mechanisms including inhibition of cancer cell proliferation, invasion and metastasis, induction of cancer cell apoptosis, epigenetic changes, and regulation of microRNA expression [20-23]. However, due to the limitation of rapid metabolism, poor solubility and low bioavailability in vivo, domestic and foreign scholars have structurally modified CU to obtain many long-acting derivatives, which obviously improved the retention time in vivo [24]. Currently, structural modification and derivatization of CU to develop its greater application potential has gradually become a research hotspot $[25,26]$. Our group structurally modified the hydroxyl group of CU in an attempt to obtain a novel target compound. Interestingly, preliminary experiments confirmed that the derivative holds the potential for adjunctive drug molding, so a novel long-acting nanodrug system encapsulating paclitaxel has been developed by using it as a carrier material.

PTX still exhibits poor aqueous solubility in cancer therapy. In this regard, solid dispersion technique, a preparation technique in which poorly soluble drugs are highly dispersed in another carrier, can effectively solve the problem of poor drug solubility [27-29]. In addition to promoting drug dissolution and improving bioavailability, this technology may also utilize the physical and chemical properties of specific carriers to control the release of the loaded drugs, thus improving the safety of certain drugs with narrow therapeutic windows [30,31]. Accordingly, this paper intends to use solid dispersion technology to prepare a novel nanodrug delivery system of PTX, which could improve the water solubility of PTX while enhancing the efficacy.

Herein, in view of the obvious side effects and other defects in the clinical application of PTX, a novel high-efficiency and low-toxicity long-acting nanodrug delivery system was developed using CUD as an encapsulating material, which was evaluated through in vitro drug release and stability investigations. The anti-tumor effect of this PTX nanoparticle against breast cancer in vitro and in vivo was investigated via cytotoxicity testing and the establishment of nude mouse tumor models. Data from hemolytic assay, acute toxicity and cumulative toxicity determination provide important evidence for this nanoparticle to 
reduce the toxicity of PTX. The drug metabolism behavior in vivo significantly prolongs the retention time of PTX in vivo, as well as increases the absorption, bioavailability and other advantages, offering a new mentality on screening PTX preparations with high effect and low toxicity.

\section{Materials And Methods}

\section{Materials}

The following reagents, drugs and chemicals were used in this study: paclitaxel (PTX, purchased from Luzhou Renkang Biotechnology Co., Ltd.), curcumin derivatives (CUD, homemade in laboratory), dipalmitoyl phosphatidylethanolamine-polyethylene glycol 2000 (DSPE-PEG2000, Lipoid GmbH, Germany), Poloxamer F68 (Shanghai Aladdin Biochemical Technology Co., Ltd.), trichloromethane (purchased from Luzhou Renkang Biotechnology Co., Ltd.) and thiazole blue (MTT, Beijing Soleboro Technology Co., Ltd.). All reagents were analytical or high-performance liquid chromatography grade.

\section{Cells}

Human breast cancer MCF-7 cells (purchased from the Cell Bank Center of Chinese Academy of Sciences) were cultured in DMEM high glucose medium containing $10 \%$ fetal bovine serum and $1 \%$ penicillin/streptomycin. The cells were cultured in a $37^{\circ} \mathrm{C}$ cell incubator containing $5 \%$ carbon dioxide humidified air.

\section{Animals}

Female BALB/c mice, aged 7 weeks old and weighing $20 \pm 3$ g, were purchased from Chengdu Dashuo Laboratory Animal Co., Ltd. (Laboratory Animal Use License: SYXK (Sichuan) 2018-065). Female Sprague Dawley (SD) mice, weighing $220 \pm 20 \mathrm{~g}$, were purchased from the Experimental Animal Center of Southwest Medical University (Laboratory Animal License: SYXK (Sichuan) 2018 -181). All mice were housed indoors with alternating light and dark cycles for $12 \mathrm{~h}$, and had free access to water and food during the adaptation period. Four-week-old female BALB/c nude mice (Laboratory Animal Production License No. SCXK (Beijing) 2016-0002) were purchased from Sparford (Beijing) Biotechnology Co., Ltd. and raised in SPF animal room of Chengbei Animal Center of Southwest Medical University.

\section{Synthesis of CUD}

After $110 \mathrm{mg}$ curcumin (CU) was dissolved in anhydrous dichloromethane, $96 \mu \mathrm{L}$ triethylamine (TEA) was added. Subsequently, 135 mg of cholesteryl chloroformate dissolved in anhydrous dichloromethane was slowly dropped to react under ice bath for $1 \mathrm{~h}$. The solvent was removed by distillation under reduced pressure after completion of the reaction, and the desired product was obtained by an extraction method. The precipitate was retained after five ultrasonic washing with methanol, and the pellet was washed with isopropanol followed by centrifugation to collect the supernatant. CUD was obtained by rotary evaporation of the supernatant to remove the solvent.

\section{Preparation of Nanoparticles}

The defined amounts of PTX, CUD, F68, DSPE-PEG2000 were accurately weighed and dissolved via appropriate amounts of trichloromethane. Lipid membranes were formed by stirring with a magnetic stirrer and evaporating the solvent at $300 \mathrm{r} / \mathrm{min}$ and $40^{\circ} \mathrm{C}$. To ensure no residual trichloromethane, the solvent was further removed with nitrogen and then cured in an oven at $40{ }^{\circ} \mathrm{C}$ for about $30 \mathrm{~min}$. The particles are uniformly dispersed by ultrasonic probe after adding deionized water for hydration. The clarified CUD-PTX-LN nano-solution was obtained by ultrasonic fragmentation at the intensity of $185 \mathrm{~W}$ for 10 min [32]. In order to long-term storage, a certain amount of sucrose (w/v) was added as a lyophilization protectant [33]. The yellow loose powder was obtained after freeze-drying for about $28 \mathrm{~h}$.

\section{Characterization of Nanoparticles Encapsulation Efficiency (EE) and Drug Loading (DL)}

Encapsulation efficiency (EE) and drug loading (DL) were measured by centrifuging freshly prepared CUD-PTX-LN nano-solution at $3000 \mathrm{r} / \mathrm{min}$ for 10 min. The free PTX was precipitated while the supernatant contained homogeneous PTX Nanoparticles. To break the nanoparticles, $4 \mathrm{~mL}$ methanol was added to $100 \mu \mathrm{L}$ of the supernatant of CUD-PTX-LN, followed by sonication for $5 \mathrm{~min}$. In addition, $100 \mu \mathrm{L}$ of uncentrifuged CUD-PTX-LN was processed as described above. The content of PTX was detected by HPLC to determine the drug content entrapped in the nano-liquid.

The encapsulation efficiency and drug loading formula are as follows:

EE $(\%)=$ Actual loading of PTX in nanoparticles /actual amount of PTX used for nanoparticles preparation $\times 100 \%$

DL $(\%)=$ Amount of PTX in nanoparticles $/$ Total amount of nanoparticles $\times 100 \%$

The chromatographic conditions are described below. Liquid chromatographic protection column: Phenomenex C18 (4.0 mm x $3.0 \mathrm{~mm})$, liquid chromatographic column: Luna $5 \mu \mathrm{m} \mathrm{C18} \mathrm{(2)} 100 \AA$ ( $4.6 \mathrm{~mm} \times 250 \mathrm{~mm}$ ), elution time: $10 \mathrm{~min}$, mobile phase: acetonitrile-water phosphate $(\mathrm{PH} 4.0)=58: 42$ (v/v), wavelength: $227 \mathrm{~nm}$, injection volume: $20 \mu \mathrm{L}$, column temperature: $30{ }^{\circ} \mathrm{C}$, flow rate: $1 \mathrm{~mL} / \mathrm{min}$.

\section{Particle Size, Zeta Potential (ZP) and Polydispersity Index (PDI)}

The CUD-PTX-LN solution was diluted to an appropriate concentration and placed into the sample pool ( $1 \mathrm{~mL})$. Particle size, polydispersity index (representing the uniformity of particle size distribution) and zeta potential were measured using a Malvern particle size analyzer at $25^{\circ} \mathrm{C}$. 
The release profile of CUD-PTX-LN was investigated with a total volume of $200 \mathrm{~mL}$ (containing $0.2 \%$ Tween-80) of PBS phosphate buffer solution as the release medium [34]. After $1 \mathrm{~mL}$ of the same concentration of CUD-PTX-LN and free PTX were separately placed into a dialysis bag (8000-14000 Da), the release trial was performed using a dissolution apparatus at $37^{\circ} \mathrm{C}$ under $120 \mathrm{r} / \mathrm{min}$. Equal amounts of release medium $(3 \mathrm{~mL})$ were withdrawn at $0.25,0.5,1,2$, $4,8,12,24,48,72,96,120,144$ and $168 \mathrm{~h}$, as well as centrifuged at $8000 \mathrm{r} / \mathrm{min}$ for $10 \mathrm{~min}$. The supernatant was subjected to HPLC detection for analysis of cumulative release.

\section{Stability}

Nanoparticles were stored at room temperature, and the appropriate amounts were collected at $24 \mathrm{~h}$. After determination of particle size, PDI, entrapment efficiency, and drug loading, the four indexes were compared with the original. The coefficient of variation (CV) was calculated to investigate the sample use stability. To guarantee the long-term preservation of the liquid formulation of the nanoparticles, the powdered solid formulation was prepared by freeze-drying technique. The CUD-PTX-LN lyophilized samples were kept in brown ampoules for 6 months, accompanied by a temperature of $25 \pm 2{ }^{\circ} \mathrm{C}$ and a relative humidity of $40 \pm 5 \%$. The same procedure described above was performed after dissolving the samples with deionized water at 1, 2, 3 and 6 months, respectively.

\section{Cytotoxicity Assay}

The toxicity of nanoparticles on MCF-7 cells was determined by thiazole blue colorimetry (MTT colorimetry). In the logarithmic growth phase, MCF-7 cells were trypsinized to prepare a cell suspension. The cells were counted and seeded into a 96 -well plate at a density of $5 \times 10^{3}$ cells/well. After the cells were cultured at $5 \% \mathrm{CO} 2$ and $37^{\circ} \mathrm{C}$ for $24 \mathrm{~h}$, five different concentrations $(5,10,20,40$ and $80 \mathrm{ug} / \mathrm{mL})$ of free PTX, CUD-PTX-LN and blank nanoparticles were added to the cultured cells, respectively. Each well was supplemented with $20 \mu \mathrm{L} \mathrm{MTT}$ solution $(5 \mathrm{mg} / \mathrm{mL})$ after $24,48,72$, and $96 \mathrm{~h}$ of continuous culture. The cultures were terminated after 3-4 h, then the medium was aspirated and $150 \mu \mathrm{L}$ dimethyl sulfoxide was added. In order to dissolve the crystals sufficiently, the mixing was shaken for $10 \mathrm{~min}$. The absorbance value (OD) of each well was measured with a microplate reader at $490 \mathrm{~nm}$ wavelength. The growth inhibition rate of drugs on tumor cells was calculated according to the formula, and then the dose-effect curve was plotted with drug concentration (ug/ $\mathrm{mL}$ ) as abscissa.

Cell growth inhibition rate $(\%)=\left[1-\left(O D\right.\right.$ experiment $\left.\left.{ }^{-} A_{\text {blank }}\right) /\left(A_{\text {control }}{ }^{-} A_{\text {blank }}\right)\right] * 100$

\section{Safety Evaluation In vitro Hemolysis}

An appropriate amount of SD rat blood was continuously stirred clockwise through a glass rod for 5 min to destroy fibrinogen. After adding $0.9 \%$ sodium chloride solution, centrifugation ( $1500 \mathrm{r} / \mathrm{min}, 10 \mathrm{~min}$ ) was performed and the supernatant was decanted. To obtain erythrocytes, the addition of $0.9 \%$ sodium chloride solution was continued and the process of centrifugation described above was repeated until the supernatant exhibited colorless. A erythrocyte suspension with a concentration of $2 \%$ was configured via supplementation with $0.9 \%$ sodium chloride solution. Different concentration groups of CUD-PTXLN (1-7), negative control (8) and positive blank control (9) were placed in the $37^{\circ} \mathrm{C}$ incubator. All groups were assessed for hemolysis after centrifugation $(1500 \mathrm{r} / \mathrm{min}, 10 \mathrm{~min})$ at 1,3 , and $5 \mathrm{~h}$, respectively. The supernatant of each sample after $5 \mathrm{~h}$ was supplemented to a 96 well plate. The microplate reader determined the absorbance (A) at the wavelength of $540 \mathrm{~nm}$, and the hemolysis rate (\%) was calculated by the following formula [35].

Hemolysis $(\%)=\left(A_{\text {sample }}-\mathrm{A}_{\text {negative }}\right) /\left(\mathrm{A}_{\text {positive }}-\mathrm{A}_{\text {negative }}\right) \times 100 \%$

\section{Acute and Cumulative Toxicity In vivo}

Sixty mice were randomly divided into six groups (10 per group) and received the following administration forms: (A) PTX at $40 \mathrm{mg} / \mathrm{kg}$, (B) CUD-PTX-LN at 40 $\mathrm{mg} / \mathrm{kg}$, (C) normal saline with the same volume as the administration group, (D) PTX at $10 \mathrm{mg} / \mathrm{kg}$, (E) CUD-PTX-LN at $10 \mathrm{mg} / \mathrm{kg}$ and (F) normal saline with the same volume as the administration group. The first three groups constituted the acute toxicity trial, and each mouse was dosed once through the abdominal cavity after being weighed. In addition, panels C, D, and E were utilized for the evaluation of cumulative toxicity. All mice were injected intraperitoneally after weighing, and the drug was administered every 3 days for 60 days.

Throughout the experiment, the status of the mice as well as the number of deaths were observed and recorded daily. On day 60 th after drug administration, plasma and tissue (heart, liver, kidney) samples were collected from three randomly selected mice in each group for analysis and detection. The blood biochemical identification of liver and kidney function is mainly manifested in plasma samples (the following parameters were measured: alanine transaminase (ALT), aspartate transaminase (AST), lactate dehydrogenase (LDH), urea (urea), creatinine (crea) and uric acid (UA)). Tissue (heart, liver, and kidney) samples were stained with hematoxylin eosin (HE) before pathological section analysis. All operating procedures were performed in accordance with the requirements of ethical regulations.

\section{Pharmacokinetics of CUD-PTX-LN}

Ten SD rats were randomly divided into two groups (five in each group), which were treated sequentially with the following dosage: $10 \mathrm{mg} / \mathrm{kg}$ of PTX and 10 $\mathrm{mg} / \mathrm{kg}$ of CUD-PTX-LN. For each rat after drug administration, cardiac blood was sampled at $0.08,0.25,0.5,1,2,4,8,12,24,48,72,96$ and $120 \mathrm{~h}$. The removed raw blood $(0.3 \mathrm{~mL})$ was centrifuged for $3 \mathrm{~min}(5000 \mathrm{rpm} / \mathrm{min})$ in a sodium heparin containing centrifuge tube, and $100 \mu \mathrm{L}$ of the supernatant plasma was collected for detection. The plasma was vortexed for $3 \mathrm{~min}$ in $0.5 \mathrm{~mL}$ of extractant (methanol: ethyl acetate $=10: 90$, v/v), and the supernatant was obtained after centrifugation at $8000 \mathrm{rpm} / \mathrm{min}$ for $3 \mathrm{~min}$. In order to ensure adequate extraction, the remaining precipitate was again added with $0.5 \mathrm{~mL}$ of extractant to repeat the extraction. The two supernatants were combined and blown dry with nitrogen at $35^{\circ} \mathrm{C}$. $200 \mu \mathrm{L}$ of the complex solvent [phosphoric acid: acetonitrile $=75: 25(\mathrm{pH} 3.5)$ ] was added and vortexed for $4 \mathrm{~min}$ until complete dissolution. After sonication for 4 min and centrifugation at $10000 \mathrm{rpm} / \mathrm{min}$ for $10 \mathrm{~min}$, the supernatant was collected and detected by HPLC. 
The chromatographic conditions are described below. Liquid chromatographic protection column: Phenomenex C18 (4.0 mm x $3.0 \mathrm{~mm})$, liquid chromatographic column: Luna $5 \mu \mathrm{m} \mathrm{C18} \mathrm{(2)} 100 \AA$ ( $4.6 \mathrm{~mm} \times 250 \mathrm{~mm})$, elution time: $10 \mathrm{~min}$, mobile phase: acetonitrile-water phosphate $(\mathrm{PH} 4.0)=65: 35$ (v/v), wavelength: $227 \mathrm{~nm}$, injection volume: $20 \mu \mathrm{L}$, column temperature: $30{ }^{\circ} \mathrm{C}$, flow rate: $0.8 \mathrm{~mL} / \mathrm{min}$.

\section{In vivo Antitumor Assessment}

The in vivo antitumor effect of PTX nanoparticles was investigated with tumor-bearing nude mice. $0.1 \mathrm{~mL}$ tumor cell suspension $\left(3 \times 10^{7}\right.$ cells $\left./ \mathrm{mL}\right)$ was inoculated into the left armpit of female BALB / tumor bearing nude mice. Thirty nude mice were randomly divided into three groups: (1) control group (normal saline), (2) $10 \mathrm{mg} / \mathrm{kg}$ PTX group, (3) $10 \mathrm{mg} / \mathrm{kg}$ CUD-PTX-LN group. All groups were administrated intraperitoneally every 3 days for 20 days.

After drug intervention on tumor-bearing nude mice, the body weight and tumor volume of all tumor-bearing nude mice were measured every 3 days. In addition, the cumulative number of deaths of tumor-bearing nude mice in each group was tallied every 5 days. The formula $V=\pi / 6\left(A B^{2}\right)$ was chosen to calculate the volume $(V)$ of the tumor, where $A$ and $B$ represent the largest and shortest vertical axes, respectively. The average body weight and tumor volume change curves of each group were plotted. After administration for 20 days, the nude mice were sacrificed and the tumor mass was removed for weighing, and the tumor inhibition rate $(I R)$ was calculated by the following formula: $I R=\left(1\right.$-tumor weight $_{\text {drug }} /$ tumor $_{\text {weight }}$ control $\left.\times 100 \%\right)$.

Tumor tissues were dehydrated with absolute ethanol after being fixed in $4 \%$ paraformaldehyde for $24 \mathrm{~h}$, and then embedded by paraffin to prepare tumor sections. Antigen retrieval of tissue sections was performed at a high temperature environment in $0.01 \mathrm{M}$ citric acid buffer ( $\mathrm{pH} 6.0$ ) for $5 \mathrm{~min}$. Sections were incubated in primary antibody for $\mathrm{KI} 67$ overnight at $4^{\circ} \mathrm{C}$, followed by dropwise biotinylated secondary antibody incubation for $30 \mathrm{~min}$ at $37^{\circ} \mathrm{C}$. Ultimately, the sections were subjected to image acquisition by microscopic camera system after color development with 3,30-diaminobenzidine (DAB) and hematoxylin staining. All experimental procedures were performed in a blinded manner.

\section{Statistical Analysis}

All data were expressed as mean \pm SEM deviation by SPSS 19.0 software. One-way ANOVA and Tukey's test were used for comparison between groups. P $\leq$ 0.05 was considered statistically significant.

\section{Results And Discussion Characterization of CUD}

CUD was characterized as follows: ${ }^{1} \mathrm{H}$-nuclear magnetic resonance (NMR) (400 MHz, Chloroform-d) $\delta 7.59\left(\mathrm{dd}, \mathrm{J}=15.9,5.1 \mathrm{~Hz},{ }^{2} \mathrm{H}\right), 7.21-7.01\left(\mathrm{~m},{ }^{6} \mathrm{H}\right), 6.98-$ $6.88\left(\mathrm{~m},{ }^{1} \mathrm{H}\right), 6.51\left(\mathrm{ddd}, \mathrm{J}=21.3,15.7,3.9 \mathrm{~Hz},{ }^{2} \mathrm{H}\right), 5.83\left(\mathrm{~d}, \mathrm{~J}=3.8 \mathrm{~Hz},{ }^{1} \mathrm{H}\right), 5.42\left(\mathrm{~s},{ }^{1} \mathrm{H}\right), 4.57\left(\mathrm{dt}, \mathrm{J}=11.4,5.8 \mathrm{~Hz},{ }^{1} \mathrm{H}\right), 3.92\left(\mathrm{dd}, \mathrm{J}=17.8,3.7 \mathrm{~Hz},{ }^{6} \mathrm{H}\right), 2.48(\mathrm{~d}, \mathrm{~J}=$ $\left.14.2 \mathrm{~Hz},{ }^{2} \mathrm{H}\right), 2.10-0.82\left(\mathrm{~m},{ }^{43} \mathrm{H}\right), 0.69\left(\mathrm{~d}, \mathrm{~J}=3.9 \mathrm{~Hz},{ }^{3} \mathrm{H}\right)$. Electrospray ionization mass spectrometry (ESI-MS) $\mathrm{m} / \mathrm{z} ; 779.54 \mathrm{metal}$ hydride $[\mathrm{M}+\mathrm{H}]^{+}$.

For the structure of CUD, see Fig. 1.

\section{Characterization of Nanoparticles}

The data of particle size, PDI, zeta potential, drug loading and entrapment efficiency of PTX nanoparticles were collated in Table 1. The suitable particle size of the prepared nanoparticle (CUD-PTX-LN) was $238.5 \pm 4.79 \mathrm{~nm}$. An appropriate particle size may impart the nanoparticles with a certain passive targeting ability, thereby increasing its distribution in tumor tissues. The PDI is $0.225 \pm 0.011$, indicating that the particles are uniformly distributed and normalized to a single peak (Fig. 2A). Zeta potential, an important indicator to evaluate the stability of nanoparticles, was measured to be $-33.8 \pm 1.26 \mathrm{mV}$. The surface of prepared nanoparticles carries a large negative charge, which causes the electrostatic repulsion between neighboring particles to increase the distance between particles, thus reducing the aggregation of nanoparticles [36].

Table 1

Physicochemical Characteristics of CUD-PTX-LN nanoparticles

\begin{tabular}{|llllll|}
\hline Formulation & Size $(\mathrm{mn})$ & PDI & ZP(mV) & EE(\%) & DL(\%) \\
\hline CUD-PTX-LN & $238.5 \pm 4.79$ & $0.225 \pm 0.011$ & $-33.8 \pm 1.26$ & $94.20 \pm 0.49$ & $10.98 \pm 0.31$ \\
\hline \multicolumn{7}{|l}{ Note: Each data point represents the mean \pm SEM $(n=3)$} \\
\hline
\end{tabular}

\section{In vitro Release Investigation}

The in vitro release profile of CUD-PTX-LN is shown in Fig. 3, and the cumulative average release over one week was $70.66 \%$, which was slightly lower than that of free PTX group. Fitting the data according to the in vitro release degree results, the in vitro release behavior of CUD-PTX-LN was more consistent with a zero-order model and its release rate did not change with time. However, the in vitro release behavior of free PTX was more similar to the first-order model. The drug in free PTX group released over $60 \%$ rapidly in $24 \mathrm{~h}$. Therefore, the nanoparticles presented the effects of sustained release and long circulation to some extent. Due to the formed nanostructured skeleton that somewhat restricted the corresponding entrapped drug, the nanoparticles relatively controlled and retarded the release of this drug [37]. Meanwhile, the above phenomenon may be related to the CUD and the excipient DSPE-PEG2000 added in the prescription.

\section{Stability}

As shown in Fig. 4A, the coefficients of variation of various indexes of PTX nanoparticles stored at room temperature for $24 \mathrm{~h}$ were all less than $10 \%$, indicating that the preparation was relatively stable. For long-term preservation, CUD-PTX-LN lyophilized powder formulation was prepared by freeze-drying 
technique to improve stability. There was no significant improvement in particle size, PDI, encapsulation efficiency, and drug loading of the CUD-PTX-LN lyophilized powder within 6 months, confirming that CUD-PTX-LN is steady when stored under suitable conditions for 6 months (Fig. 4B).

\section{Cytotoxicity Evaluation}

Both free PTX and CUD-PTX-LN inhibited the growth of breast cancer cells (MCF-7) to some extent in a concentration - and time-dependent manner (Fig. 5). Before $48 \mathrm{~h}, \mathrm{PTX}$ tended to be slightly better inhibited than CUD-PTX-LN, but the inhibition rate of CUD-PTX-LN is at least $10 \%$ higher than that of PTX at $72 \mathrm{~h}$ and $96 \mathrm{~h}$. This outcome might be affected via the sustained release effect of CUD-PTX-LN, and also coincided with the rule of previous in vitro drug release assays of CUD-PTX-LN. Besides that, the inhibition ratio caused by CUD-PTX-LN was significantly higher than that of PTX group at low and medium concentrations $(20,40 \mu \mathrm{g} / \mathrm{mL})$. This phenomenon confirmed that CUD-PTX-LN is characterized by synergism and low toxicity.

As far as the normal hepatocyte L02 is concerned, the inhibitory effect of PTX on L02 was higher than that of CUD-PTX-LN at various concentrations and time points (Fig. 6). Especially at $96 \mathrm{~h}$, the inhibition rate of cell growth by free PTX at a concentration of $10 \mu \mathrm{g} / \mathrm{mL}$ was $40.13 \%$ higher than that of CUD-PTX-LN. This illustrates that the safety profile of CUD-PTX-LN is superior to that of PTX.

\section{In vitro Hemolysis}

Panels 7 exhibit hemolysis in different CUD-PTX-LN concentration groups as well as hemolysis rates. CUD-PTX-LN at low concentration (groups 1-2) did not cause any hemolysis, either in terms of appearance or hemolysis rate. Similarly, the appearance of CUD-PTX-LN in the medium and high concentration groups (groups 3-7) did not exhibit a distinct red color, and its hemolysis did not exceed 5\%. Therefore, this result suggested that CUD-PTX-LN did not cause severe hemolysis phenomenon. Clinically to improve the solubility of free PTX, ethanol and hydrogenated castor oil are often chosen as mixed lysosomes, but this mixed vehicle in turn can further aggravate the hemolytic reaction [38]. CUD-PTX-LN prepared by solid dispersion technique not only better controlled the hemolytic side reaction of PTX, but also greatly improved the aqueous solubility [39]. The associated toxic side reactions from this mixed vehicle are also absent.

\section{In vivo Acute and Cumulative Toxicity}

\section{Mouse Mortality}

The conclusion of acute toxicity trials is available in Table 2, and 10 mice all died within $5 \mathrm{~h}$ after a single intraperitoneal injection of free PTX at $40 \mathrm{mg} / \mathrm{kg}$ (Fig. 8A). Since free PTX and the mixed vehicle (hydrogenated castor oil and absolute ethanol) caused severe allergic and cardiotoxicity [40]. However, seven days after a single intraperitoneal injection of $40 \mathrm{mg} / \mathrm{kg}$ CUD-PTX-LN, the number of deaths in mice was four. Although the activity of the mice in this group decreased compared with that before injection, there was no respiratory acceleration and convulsion phenomenon. The 6 mice that subsequently survived also gradually returned to normal, with an increase in no new dead mice between 7 and 60 days. In the cumulative toxicity assays, three mice died within 7 days in the free PTX group, and all the mice in this group died within 60 days (Fig. 8B). Comparatively, the CUD-PTX-LN nanoparticles group had only two numbers of mouse deaths throughout the course of the experiment.

Table 2

Effects on serum biochemical parameters of mice after intraperitoneal administration in different experimental groups. A single intraperitoneal injection of $\mathrm{P} 7$ CUD-PTX-LN (B) at 40mg/kg and an equal volume of saline (C). Intraperitoneal injection every three days for 60 days: PTX (D), CUD-PTX-LN (E) at 10mg/kg a equal volume of saline $(E)$. $(n=3)$.

\begin{tabular}{|c|c|c|c|c|c|c|c|c|c|c|c|c|c|c|c|c|}
\hline \multirow{2}{*}{$\begin{array}{l}\begin{array}{l}\text { Biochemical } \\
\text { indicators }\end{array} \\
\text { ALT } \\
(\mathrm{U} / \mathrm{L})\end{array}$} & \multicolumn{3}{|c|}{ Group B } & \multicolumn{3}{|c|}{ Group C } & \multicolumn{3}{|c|}{ Group D } & \multicolumn{3}{|c|}{ Group E } & \multicolumn{3}{|c|}{ Group F } & \multirow{2}{*}{$\begin{array}{l}\text { Refe } \\
\text { ranç } \\
33.0 \\
98.7\end{array}$} \\
\hline & 80.4 & 69.7 & 73.5 & 51.1 & 63.8 & 47.1 & 46.8 & 107.5 & 113.2 & 83.6 & 60.7 & 79.1 & 66.4 & 74.8 & 50.8 & \\
\hline $\begin{array}{l}\text { AST } \\
(\mathrm{mmol} / \mathrm{L})\end{array}$ & 2.68 & 6.04 & 3.55 & 5.42 & 4.82 & 6.04 & 4.42 & 8.33 & 6.06 & 4.78 & 7.94 & 6.23 & 2.69 & 5.04 & 3.43 & $\begin{array}{l}2.00 \\
7.70\end{array}$ \\
\hline $\begin{array}{l}\text { LDH } \\
(\mathrm{U} / \mathrm{L})\end{array}$ & 97.8 & 166.7 & 424.6 & 439.7 & 368.5 & 347.2 & 567.9 & 632.5 & 608.4 & 369.2 & 406.7 & 516.3 & 328.5 & 269.7 & 440.3 & $\begin{array}{l}101 . \\
514 .\end{array}$ \\
\hline $\begin{array}{l}\text { UREA } \\
(\mu \mathrm{mol} / \mathrm{L})\end{array}$ & 30.9 & 49.7 & 45.8 & 58.2 & 99.2 & 73.6 & 112.1 & 93.5 & 126.0 & 48.0 & 78.8 & 56.7 & 46.2 & 57.0 & 81.6 & $\begin{array}{l}22.0 \\
97.0\end{array}$ \\
\hline $\begin{array}{l}\text { CREA } \\
(\mu \mathrm{mol} / \mathrm{L})\end{array}$ & 78.2 & 20.5 & 79.2 & 59.9 & 39.8 & 66.4 & 144.7 & 196.5 & 152.8 & 69.2 & 59.3 & 102.8 & 79.6 & 102.7 & 94.5 & $\begin{array}{l}20.0 \\
120 .\end{array}$ \\
\hline UA & 116.5 & 205.3 & 103.0 & 108.4 & 97.4 & 122.6 & 90.8 & 246.7 & 185.3 & 90.4 & 118.5 & 176.2 & 142.5 & 166.2 & 104.9 & $\begin{array}{l}69.5 \\
210 .\end{array}$ \\
\hline
\end{tabular}

Abbreviations:

ALT, alanine aminotransferase; AST, aspartate aminotransferase; ALP, alkaline phosphatase; CREA, creatinine; UA, uric acid.

\section{Blood Biochemical Parameters}

The whole mice of the PTX acute toxicity group (group A) were weak in vital signs and died soon after administration, the corresponding plasma samples could not be collected. Comparison of biochemical parameters of mice in the CUD-PTX-LN acute toxicity group with that of the control group, only one mouse had an LDH value slightly below the reference range. In addition, the CREA index of another mouse is slightly lower than the reference range but has no clinical 
guiding significance (Table 2) [41]. In general, only one or two biochemical indexes were abnormal in the same group of mice, confirming to some extent that liver and kidney injury brought by CUD-PTX-LN to mice was limited. In the cumulative toxicity assay, the relevant indicators of each mice in PTX group revealed high values compared with the control group. Elevations in ALT, AST and LDH imply that metabolic processes such as normal biochemical enzymology in the liver are affected [42]. Values of UREA, CREA, UA above the upper limit of the reference range also suggested damage to the kidneys and affected normal physiological and biochemical processes [43]. Conversely, the biochemical parameters of all mice were normal in the CUD-PTX-LN group.

\section{Pathology Slides}

The slice images of the heart, liver, and kidney tissues in both the PTX acute toxicity group (group A) and CUD-PTX-LN acute toxicity group (group B) indicated some degree of necrosis in the corresponding tissues (Fig. 9A). However, the deformation, necrosis and vascular damage of myocardial fibers in the heart of group A were more obvious than those of group B. In group A, hepatocytes exhibited more nuclear division and enlarged liver fibers. Similarly, the degree of degeneration and necrosis of cardiac muscle fibers in the heart of group A was more severe than that of group B. Therefore, CUD-PTX-LN caused lower damage to tissues in the heart, liver, and kidney than free PTX in acute toxicity assays.

The results of pathological sections of mouse tissues (heart, liver, and kidney) for the cumulative toxicity trials are shown in Fig. 9B. The tissues of mice in the CUD-PTX-LN cumulative toxicity group (group E) did not reveal apparent lesions and necrosis. After continuous administration of free PTX, some damage of cells and muscle fibers appeared in the tissues of the heart, liver and kidney parts of mice. Therefore, CUD-PTX-LN exhibits a higher safety index accompanied by less cumulative toxicity than free PTX. This behavior also further verified that the toxicity caused by CUD-PTX-LN might be more tolerable than PTX during the course of long-term medication.

\section{Pharmacokinetics}

The pharmacokinetic behaviors of free PTX and CU-PTX-LN in SD rats over $120 \mathrm{~h}$ were delineated by a concentration-time curve (Fig. 10). The relevant pharmacokinetic parameters were also calculated (Table 3). Significantly higher plasma PTX concentrations observed in the rats subjected to CUD-PTX-LN than in this exposed to free PTX. Free PTX took less time to complete metabolism in vivo than nanoparticles. The area under the curve (AUC ${ }_{0-t}$ ) of CUD-PTXLN was $14.72 \mathrm{mg} / \mathrm{L} \times \mathrm{h}$, which was greater than that of free PTX group $(\mathrm{P}<0.01)$. Table 3 also shows that the half-life $\left(\mathrm{t}_{1 / 2}\right)$ of CUD-PTX-LN exhibited a 4-fold advantage over free PTX. However, its clearance (CL) was 1 / 7 of free PTX group. The differences in the values of these indexes confirmed that CUD-PTX-LN significantly prolonged the drug residence time in the blood, maintained a higher blood drug concentration for a long time and increased bioavailability.

Table 3

pharmacodynamic parameters in rats following injection administration of CUD-PTX-LN and PTX.

\begin{tabular}{|c|c|c|c|}
\hline \multirow[t]{2}{*}{ parameter } & \multirow[t]{2}{*}{ Units } & \multicolumn{2}{|l|}{$10 \mathrm{mg} / \mathrm{kg}$} \\
\hline & & PTX & CUD-PTX-LN \\
\hline $\operatorname{AUC}_{(0-t)}$ & $\mathrm{mg} / \mathrm{L}^{\star \mathrm{h}}$ & $2.381 \pm 0.276$ & $14.716 \pm 1.985^{\star \star *}$ \\
\hline $\operatorname{AUC}_{(0-\infty)}$ & $\mathrm{mg} / \mathrm{L}^{* h}$ & $4.368 \pm 0.365$ & $33.829 \pm 4.432^{\star \star}$ \\
\hline $\mathrm{MRT}_{(0-t)}$ & $\mathrm{h}$ & $4.94 \pm 0.653$ & $38.444 \pm 2.983^{\star *}$ \\
\hline $\operatorname{MRT}_{(0-\infty)}$ & $\mathrm{h}$ & $15.338 \pm 1.892$ & $15.338 \pm 2.076$ \\
\hline$t_{1 / 2 z}$ & $\mathrm{~h}$ & $10.991 \pm 0.773$ & $44.293 \pm 3.012^{\star \star}$ \\
\hline $\mathrm{T}_{\max }$ & $\mathrm{h}$ & $0.25 \pm 0.02$ & $0.61 \pm 0.07 * \star$ \\
\hline $\mathrm{V}_{\mathrm{z}}$ & $\mathrm{L} / \mathrm{kg}$ & $54.467 \pm 8.532$ & $37.46 \pm 4.82^{\star *}$ \\
\hline $\mathrm{CL}_{\mathrm{z}}$ & $\mathrm{L} / \mathrm{h} / \mathrm{kg}$ & $3.434 \pm 0.312$ & $0.443 \pm 0.052^{\star \star}$ \\
\hline
\end{tabular}

\section{In vivo Antitumor Investigation}

The tumors grew in different proportions after the initiation of drug administration to the nude mice in each group. The tumor volume of the model group increased more significantly, reaching $1800 \mathrm{~mm}^{3}$ after 20 days of administration (Fig. 11A). Interestingly, the tumor growth rate of free PTX group and CUDPTX-LN group was retarded, especially in CUD-PTX-LN group. After 13 days of administration, the tumor volume in this group of nude mice was only 750 mm ${ }^{3}$ and was subsequently maintained at a level of $750 \mathrm{~mm}^{3}$ until the completion of the trial. The average weight of tumors in the CUD-PTX-LN group after nude mice were sacrificed was also the lightest (Fig. 11F). The tumor volume was significantly suppressed in nude mice after the initiation of intervention by free PTX. However, tumor volume followed another head up growth trend after 13 days, reaching $1000 \mathrm{~mm}^{3}$ by 20 days. Therefore, CUD-PTX-LN effectively inhibited MCF-7 tumor growth in nude mice, and the efficacy was superior to that of free PTX. In addition, the static tumor inhibition rate results also confirmed the superior antitumor efficacy of CUD-PTX-LN $(44.34 \%)$ over PTX $(32.93 \%)(P<0.01)$.

After the in vivo antitumor evaluation was completed, Ki-67 immunohistochemical staining was employed to observe the anti-proliferation efficacy of CUDPTX-LN. The weakest immunohistochemical reaction (<20\%) was observed in the CUD-PTX-LN group (Fig. 11D). H \& E staining plots exhibited various degrees of necrotic areas in all treatment groups except the saline control group. Approximately one-half of cancer cell necrosis was detected when treated 
with CUD-PTX-LN. However, free PTX corresponded to a cancer cell necrosis rate of $25 \%$. These phenomena were consistent with the above conclusions of Ki67 IHC staining analysis.

Although the average body weight of nude mice in each group decreased after administration, the least decrease was in the CUD-PTX-LN group. There was no significant difference in the average body weight of nude mice after 20 days of drug administration in this group compared to the beginning (Fig. 11B). Free PTX resulted in the death of three nude mice throughout the test, which may be related to the combined toxicity caused by PTX as well as the mixed vehicle (ethanol and hydrogenated castor oil) [44]. In contrast, CUD-PTX-LN nanoparticles exhibited no obvious side effects, indicating that they were safer than free PTX.

\section{Dicussion}

The structural modification of curcumin to some extent solves its disadvantages of rapid metabolism and low bioavailability in clinical applications [24, 45]. However, curcumin derivatives obtained by structural modification mostly exert antitumor and multifaceted activities directly as drugs, and there is a lack of exploration of other application potentials [46]. The highlight of this study is that novel derivatives synthesized with curcumin as the parent not directly coloaded paclitaxel as a pharmaceutical chaperone, but rather as carrier materials to develop a drug delivery system containing paclitaxel. Nanoparticles with long-acting functionality were obtained by combining the properties of this derivative and the anticancer activity of paclitaxel, while enhancing the efficacy of PTX and reducing toxicity.

Solid dispersion technology, applied to some water-insoluble carriers or poorly soluble materials as carriers of drugs hinders the release of drugs to achieve the purpose of sustained or controlled release [47]. This methodology was used to prepare the CUD-PTX-LN nanodrug delivery system since it is simple, reliable and expected to quantify the production. In the subsequent in vitro release degree trials, the model fitting results of the release profiles also confirmed that this drug delivery system exhibited a certain sustained-release effect. In addition, the above conclusion might also be related to the carrier CUD added in the formulation as well as the excipient dspe-peg2000[48]. In terms of stability, normal temperature had an effect on the particle size, encapsulation efficiency and drug loading of CUD-PTX-LN, especially beyond $24 \mathrm{~h}$. However, this problem can be effectively solved by lyophilization and its critical performance can remain stable over 6 months. Therefore, the whole drug delivery system is reliable, which is conducive for further antitumor research and future medical applications.

Cytotoxicity test suggested that CUD-PTX-LN presented good selectivity and sensitivity for breast cancer cells MCF-7. Further analysis found that at the same low and medium concentrations, this advantage of cell growth inhibition rate caused by CUD-PTX-LN was more prominent than that by PTX, and this advantage was further strengthened with time. Considering the inhibition rate to normal hepatocytes, the damage caused by CUD-PTX-LN is weaker than that of PTX, which manifests its safety to some extent.

Safety is an important consideration for preclinical design of antitumor drug delivery systems. Favorable long-term safety evaluation of drug delivery systems can accelerate the translation of preclinical studies into clinical applications [49]. Hemolysis, hepatotoxicity and cardiotoxicity were reported as adverse effects by the clinical PTX injection test [50]. However, injection of CUD-PTX-LN into rats hardly caused hemolysis. CUD-PTX-LN nanoparticles effectively dispersed PTX and prevented its excessive accumulation, which was revealed by pathological examination that no severe organ damage was found with the treatment of CUD-PTX-LN compared with free PTX. These behaviors also indirectly confirmed that mice injected with CUD-PTX-LN died less than the free PTX group, whether in acute toxicity or cumulative toxicity experiments. Meanwhile, it was determined that $10 \mathrm{mg} / \mathrm{kg}$ of CUD-PTX-LN was safe when administered chronically.

The bioavailability of PTX in clinic is not high [51]. However, CUD-PTX-LN prolonged the retention time of PTX in vivo, decreased the clearance rate and improved the bioavailability. Furthermore, this nanoparticle enhanced the solubility and permeability of PTX. In this way, it may be possible to improve the relative effectiveness of the drug against breast cancer in vitro and in vivo. The results of the in vivo antitumor investigation indicated that CUD-PTX-LN controlled tumor growth better than free PTX. Tumor staining with Ki-67 antigen revealed that CUD-PTX-LN suppressed the proliferation of breast cancer cells. This finding was consistent with the cytotoxicity results in MCF-7 cells. The overall efficacy of the CUD-PTX-LN nanodrug delivery system against breast cancer can be explained by the fact of its improved pharmacokinetics.

\section{Conclusions}

In the present study, a novel structural curcumin derivative, CUD, as a carrier material was used in a novel nanosystem for drug delivery. We successfully prepared a novel long-acting nanodrug delivery system of paclitaxel with high efficiency and low toxicity. The preparation exhibited high entrapment efficiency and stability, and its particle size was suitable and well distributed. In vivo anti-tumor study demonstrated that the PTX nanoparticles (CUD-PTX-LN) exerted a better inhibitory effect on the growth of subcutaneous breast cancer than free paclitaxel. Furthermore, the effect was validated in cytotoxicity experiments. CUD-PTX-LN significantly improved the pharmacokinetic behavior of PTX, which involves prolonging the retention time of the drug in the body, improving its absorption and bioavailability. Most importantly, hemolytic, acute toxicity, and cumulative toxicity trials demonstrated that CUD-PTX-LN further reduced the toxicity of paclitaxel, which was consistent with the cytotoxicity evaluation of the two drugs against normal L02 hepatocytes. Therefore, CUD-PTX-LN has great potential in improving the treatment of breast cancer, thereby improving the prognosis and quality of life of patients.

\section{Declarations}

\section{Acknowledgments}

Not applicable. 


\section{Authors' contributions}

$\mathrm{MZ}$ and $\mathrm{CP}$ designed the research, performed experiments and participated in data analysis. PG, HS performed experiments, participated in data analysis and wrote the manuscript. YZ and YG characterized the Nanoparticles. KL, WZ performed in vitro and in vivo experiments and analyzed the data. YW and LZ are the guarantor of this work and provided technical guidance. All authors reviewed and edited the manuscript. All authors read and approved the final manuscript.

\section{Funding}

This study was supported by the Cooperation Projectors of Chunhui Plan of the Ministry of Education of China (N0. 2020-703), the Youth Science and Technology Innovation Research Ream(No.2021JDTD0008) and basic research fund (No.2020YJ0336, 2020YJ0373) of the Science and Technology Department of Sichuan province of China, the Joint Fund of Luzhou City and Southwest Medical University [No.2019LZXNYDZ07], the Science and Technology Fund of Luzhou science and technology and Human Resources Bureau (No. 2019-SYF-35), Science and Technology Innovation Team from Jiucheng Science and Technology Talent Cultivation Plan in Luzhou City (No. 2019-1).

\section{Availability of data and materials}

All data generated or analyzed during this study are included in this manuscript and its Additional file.

\section{Declarations}

The authors report no conflicts of interest in this work.

\section{Ethics approval and consent to participate}

All animal studies (including the mice euthanasia procedure) were done in compliance with the regulations and guidelines of Southwest Medical University institutional animal care and conducted according to the AAALAC and the IACUC guidelines

\section{Consent for publication}

Not applicable.

\section{Competing interests}

The authors declare that they have no competing interests.

\section{Author details}

${ }^{1}$ Central Nervous System Drug Key Laboratory of Sichuan Province, Southwest Medical University, Luzhou, Sichuan, 646000, PR China. ${ }^{2}$ Department of Pharmacy, The Affiliated Hospital of Southwest Medical University, Luzhou, Sichuan, 646000 , PR China. ${ }^{3}$ Department of Clinical Pharmacy, The Affiliated Hospital of Traditional Chinese Medicine of Southwest Medical University; Luzhou, Sichuan, 646000, PR China. ${ }^{4}$ Institute of medicinal chemistry of Chinese Medicine, Chongqing Acad- emy of Chinese MateriaMedica, Chongqing 400065 China ${ }^{5}$ Department of Spinal Surgery, The Affiliated Hospital of Traditional Chinese Medicine of Southwest Medical University; Luzhou, Sichuan, 646000, PR China. ${ }^{6}$ Luzhou Key Laboratory of Research and Development of Traditional Chinese Medicine for Chronic Diseases, The Affiliated Hospital of Traditional Chinese Medicine of Southwest Medical University; Luzhou, Sichuan, 646000, PR China. ${ }^{7}$ Central Nervous System Drug Key Laboratory of Sichuan Province, Southwest Medical University, Luzhou, Sichuan, 646000, PR China

\section{References}

1. Chong Y, Jin W. A Physical mechanism and global quantification of breast cancer. Plos One. 2016;11(7):422-6.

2. Ghoncheh M, Pournamdar Z, Salehiniya H. Incidence and mortality and epidemiology of breast cancer in the world. Asian Pac J Cancer Prev. 2016;17(S3):43-6.

3. Abu Samaan TM, Samec M, Liskova A, Kubatka P, Büsselberg D. Paclitaxel's mechanistic and clinical effects on breast cancer. Biomolecules. 2019;9(12):789.

4. Au YC, Co NN, Tsuruga T, et al. Exosomal transfer of stroma-derived miR21 confers paclitaxel resistance in ovarian cancer cells through targeting APAF1. Nat Commun. 2016;7:150-62

5. Della Corte L, Barra F, Foreste V, et al. Advances in paclitaxel combinations for treating cervical cancer. Expert Opin Pharmacother. 2020;21(6):663-77.

6. Cui H, Arnst K, Miller DD, Li W. Recent advances in elucidating paclitaxel resistance mechanisms in non-small cell lung cancer and strategies to overcome drug resistance. Curr Med Chem. 2020;27(39):6573-95.

7. Gu W, Chen J, Patra P, et al. Nanoformulated water-soluble paclitaxel to enhance drug efficacy and reduce hemolysis side effect. J Biomater Appl. 2017;32(1):66-73.

8. Björn N, Jakobsen Falk I, Vergote I, Gréen H. ABCB1 variation affects myelosuppression, progression-free survival and overall survival in paclitaxel/carboplatin-treated ovarian cancer patients. Basic Clin Pharmacol Toxicol. 2018;123(3):277-87. 
9. Green H, Khan MS, Ingrid JF, et al. Impact of CYP3A5*3 and CYP2C8-HapC on paclitaxel/carboplatin-induced myelosuppression in patients with ovarian cancer. J Pharm Sci. 2011;100(10):4205-9.

10. Parisi A, Palluzzi E, Cortellini A, et al. First-line carboplatin/nab-paclitaxel in advanced ovarian cancer patients, after hypersensitivity reaction to solventbased taxanes: a single-institution experience. Clin Transl Oncol. 2020;22(1):158-62.

11. Otani IM, Lax T, Long AA, Slawski BR, Camargo CA Jr, Banerji A. Utility of risk stratification for paclitaxel hypersensitivity reactions. J Allergy Clin Immunol Pract. 2018;6(4):1266-73.

12. Ma Y, Mou Q. D Yan, et al. Engineering small molecule nanodrugs to overcome barriers for cancer therapy. View. 2020:20200062.

13. Zhou M, Xie Y, Xu S, et al. Hypoxia-activated nanomedicines for effective cancer therapy. Eur J Med Chem. 2020;195:112274.

14. Fan Q, Li L, Xue H, et al. Precise control over kinetics of molecular assembly: production of particles with tunable sizes and crystalline forms. Angew Chem Int Ed Engl. 2020;59(35):15141-6.

15. Dubey RD, Saneja A, Gupta PK, Gupta PN. Recent advances in drug delivery strategies for improved therapeutic efficacy of gemcitabine. Eur J Pharm Sci. 2016;93:147-62.

16. Zhang W, Li C, Shen C, et al. Prodrug-based nano-drug delivery system for co-encapsulate paclitaxel and carboplatin for lung cancer treatment. Drug Deliv. 2016;23(7):2575-80.

17. Deng W, Qiu J, Wang S, et al. Development of biocompatible and VEGF-targeted paclitaxel nanodrugs on albumin and graphene oxide dual-carrier for photothermal-triggered drug delivery in vitro and in vivo. Int J Nanomedicine. 2018;13:439-53.

18. Choi SK. Photoactivation strategies for therapeutic release in nanodelivery systems. Adv Ther. 2020.

19. Mishra V, Bansal KK, Verma A, et al. Solid lipid nanoparticles: emerging colloidal nano drug delivery systems. Pharmaceutics. 2018;10(4):191.

20. Wang L, Wang C, Tao Z, et al. Curcumin derivative WZ35 inhibits tumor cell growth via ROS-YAP-JNK signaling pathway in breast cancer. J Exp Clin Cancer Res. 2019;38(1):460.

21. Coker-Gurkan A, Celik M, Ugur M, et al. Curcumin inhibits autocrine growth hormone-mediated invasion and metastasis by targeting NF-KB signaling and polyamine metabolism in breast cancer cells. Amino Acids. 2018;50(8):1045-69.

22. Mortezaee K, Salehi E, Mirtavoos-Mahyari H, et al. Mechanisms of apoptosis modulation by curcumin: Implications for cancer therapy. J Cell Physiol. 2019;234(8):12537-50.

23. Hassan FU, Rehman MS, Khan MS, et al. Curcumin as an alternative epigenetic modulator: mechanism of action and potential effects. Front Genet. 2019;10:514.

24. Rodrigues FC, Anil Kumar NV, Thakur G. Developments in the anticancer activity of structurally modified curcumin: An up-to-date review. Eur J Med Chem. 2019;177:76-104.

25. Kim DC, Ku SK, Bae JS. Anticoagulant activities of curcumin and its derivative. BMB cancer. 2012;45(4):221-6.

26. Aziz MT, El-Asmar MF, Rezq AM, et al. Effects of a novel curcumin derivative on insulin synthesis and secretion in streptozotocin-treated rat pancreatic islets in vitro. Chinese Medicine. 2014;9(1):3.

27. Ma Z, Wang N, He H, Tang X. Pharmaceutical strategies of improving oral systemic bioavailability of curcumin for clinical application. J Control Release. 2019;316:359-80.

28. Mbese Z, Khwaza V, Aderibigbe BA. Curcumin and its derivatives as potential therapeutic agents in prostate, colon and breast cancers. Molecules. 2019;24(23):4386.

29. Khorasani MY, Langari H, Sany SBT, Rezayi M, Sahebkar A. The role of curcumin and its derivatives in sensory applications. Mater Sci Eng C Mater Biol Appl. 2019;103:109792.

30. Nakamae I, Morimoto T, Shima H, et al. Curcumin derivatives verify the essentiality of ros upregulation in tumor suppression. Molecules. 2019;24(22):4067.

31. Komal K, Chaudhary S, Yadav P, Parmanik R, Singh M. The therapeutic and preventive efficacy of curcumin and its derivatives in esophageal cancer. Asian Pac J Cancer Prev. 2019;20(5):1329-37.

32. Ramadass SK, Anantharaman NV, Subramanian S, et al. Paclitaxel/Epigallocatechin gallate coloaded liposome: A synergistic delivery to control the invasiveness of MDA-MB-231 breast cancer cells. Colloids Surf B Biointerfaces. 2015;125:65-72.

33. Kordowska WM, Waśko A, Berecka MP, et al. Spirulina enhances the viability of Lactobacillus rhamnosus E/N after freeze-drying in a protective medium of sucrose and lactulose. Lett Appl Microbiol. 2011;53(1):79-83.

34. Huang Y, Wei Y, Yang H, et al. A 5-fluorouracil-loaded floating gastroretentive hollow microsphere: development, pharmacokinetic in rabbits, and biodistribution in tumor-bearing mice. Drug Des Devel Ther. 2016;10:997-1008.

35. Wang G, Lei Z, Zhong Q, et al. Enrichment of caffeic acid in peanut sprouts and evaluation of its in vitro effectiveness against oxidative stress-induced erythrocyte hemolysis. Food Chem. 2017;217:332-41.

36. Feng X, Pi C, Fu S, et al. Combination of curcumin and paclitaxel liposomes exhibits enhanced cytotoxicity towards A549/A549-T cells and unaltered pharmacokinetics. J Biomed Nanotechnol. 2020;16(8):1304-13.

37. Peng LH, Wei W, Shan YH, et al. Sustained release of piroxicam from solid lipid nanoparticle as an effective anti-inflammatory therapeutics in vivo. Drug Dev Ind Pharm. 2016;43(1):1-25.

38. Gu W, Chen J, Patra P, et al. Nanoformulated water-soluble paclitaxel to enhance drug efficacy and reduce hemolysis side effect. J Biomater Appl. 2017;32(1):66-73.

Page 10/18 
39. Shanmugam S, Im HT, Sohn YT, et al. Enhanced oral bioavailability of paclitaxel by solid dispersion granulation. Drug Dev Ind Pharm. 2015;41(11):186476.

40. Campos FC, Victorino VJ, Martins-Pinge MC, et al. Systemic toxicity induced by paclitaxel in vivo is associated with the solvent cremophor EL through oxidative stress-driven mechanisms. Food Chem Toxicol. 2014;68(6):78-86.

41. Wang F, Jiang YS, Liu F. The influence of mutant lactobacilli on serum creatinine and urea nitrogen concentrations and renal pathology in $5 / 6$ nephrectomized rats. Ren Fail. 2016;38(9):1441-7.

42. Awhin EP, Jeroh E, Anigboro AA, et al. Effect of chronic consumption of Piliostigma thonningii on activities of alanine aminotransferase and aspartate aminotransferase in serum and liver in Rattus novergicus. Pak J Biol Sci. 2013;16(24):2062-5.

43. Zhao H, Hong J, Yu X, et al. Oxidative stress in the kidney injury of mice following exposure to lanthanides trichloride. Chemosphere. 2013;93(6):875-84.

44. Selting KA, Bechtel SM, Espinosa J, et al. Evaluation of intravenous and subcutaneous administration of a novel, excipient-free, nanoparticulate formulation of paclitaxel in dogs with spontaneously occurring neoplasia. Vet Comp Oncol. 2018;16(4):650-7.

45. Zhao S, Pi C, Ye Y, Zhao L, Wei Y. Recent advances of analogues of curcumin for treatment of cancer. Eur J Med Chem. 2019;180:524-35.

46. Khezri K, Maleki Dizaj S, Rahbar Saadat Y, et al. Osteogenic differentiation of mesenchymal stem cells via curcumin-containing nanoscaffolds. Stem Cells Int. 2021;2021:1520052.

47. Tran PHL, Lee BJ, Tran TTD. Fast-dissolving solid dispersions for the controlled release of poorly watersoluble drugs. Curr Pharm Des. 2021;27(12):1498506.

48. Roque MC, Franco MS, Vilela JMC, et al. Development of long-circulating and fusogenic liposomes co-encapsulating paclitaxel and doxorubicin in synergistic ratio for the treatment of breast cancer. Curr Drug Deliv. 2019;16(9):829-38.

49. Guo P, Pi C, Zhao S, et al. Oral co-delivery nanoemulsion of 5-fluorouracil and curcumin for synergistic effects against liver cancer. Expert Opin Drug Deliv. 2020;17(10):1473-84.

50. Ashrafizadeh M, Zarrabi A, Hashemi F, et al. Curcumin in cancer therapy: A novel adjunct for combination chemotherapy with paclitaxel and alleviation of its adverse effects. Life Sci. 2020;256:117984.

51. Liu Z, Zheng X, Lv J, et al. Pharmacokinetic synergy from the taxane extract of Taxus chinensis improves the bioavailability of paclitaxel. Phytomedicine. 2015;22(5):573-8.

\section{Figures}




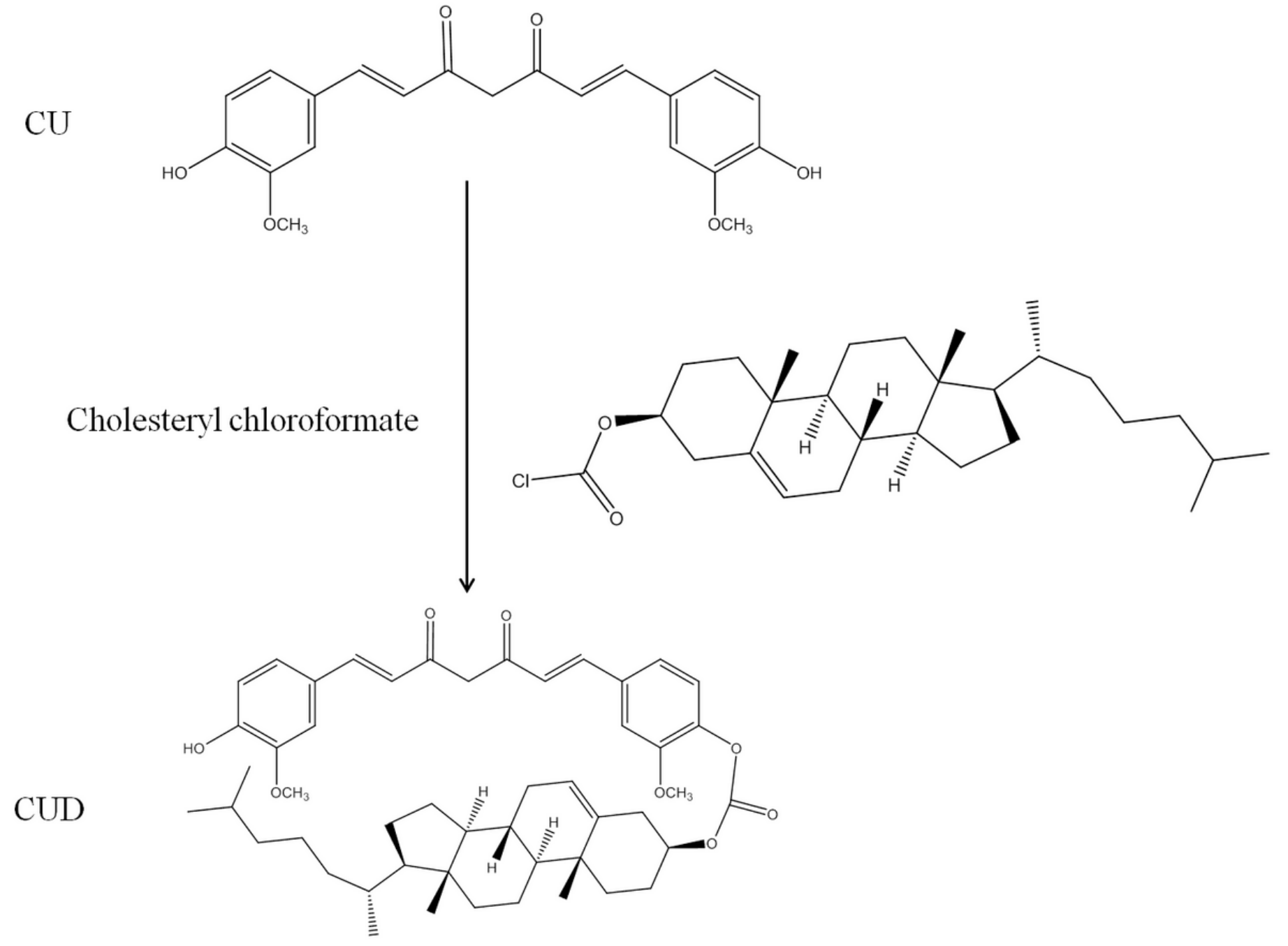

Figure 1

Caption not included with this version 
Statistics Graph (1 measurements)

A

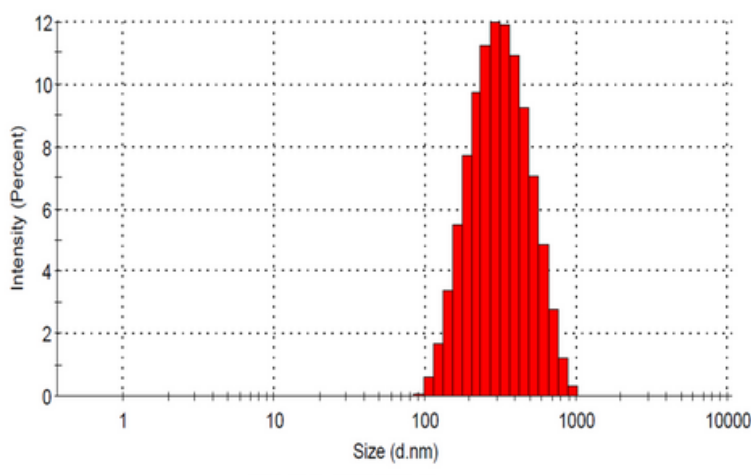

Zeta Potential Distribution

B

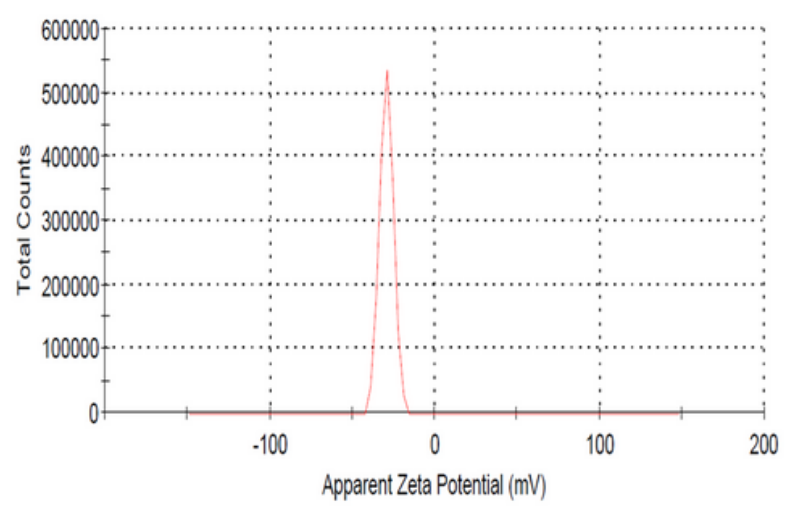

Figure 2

Caption not included with this version

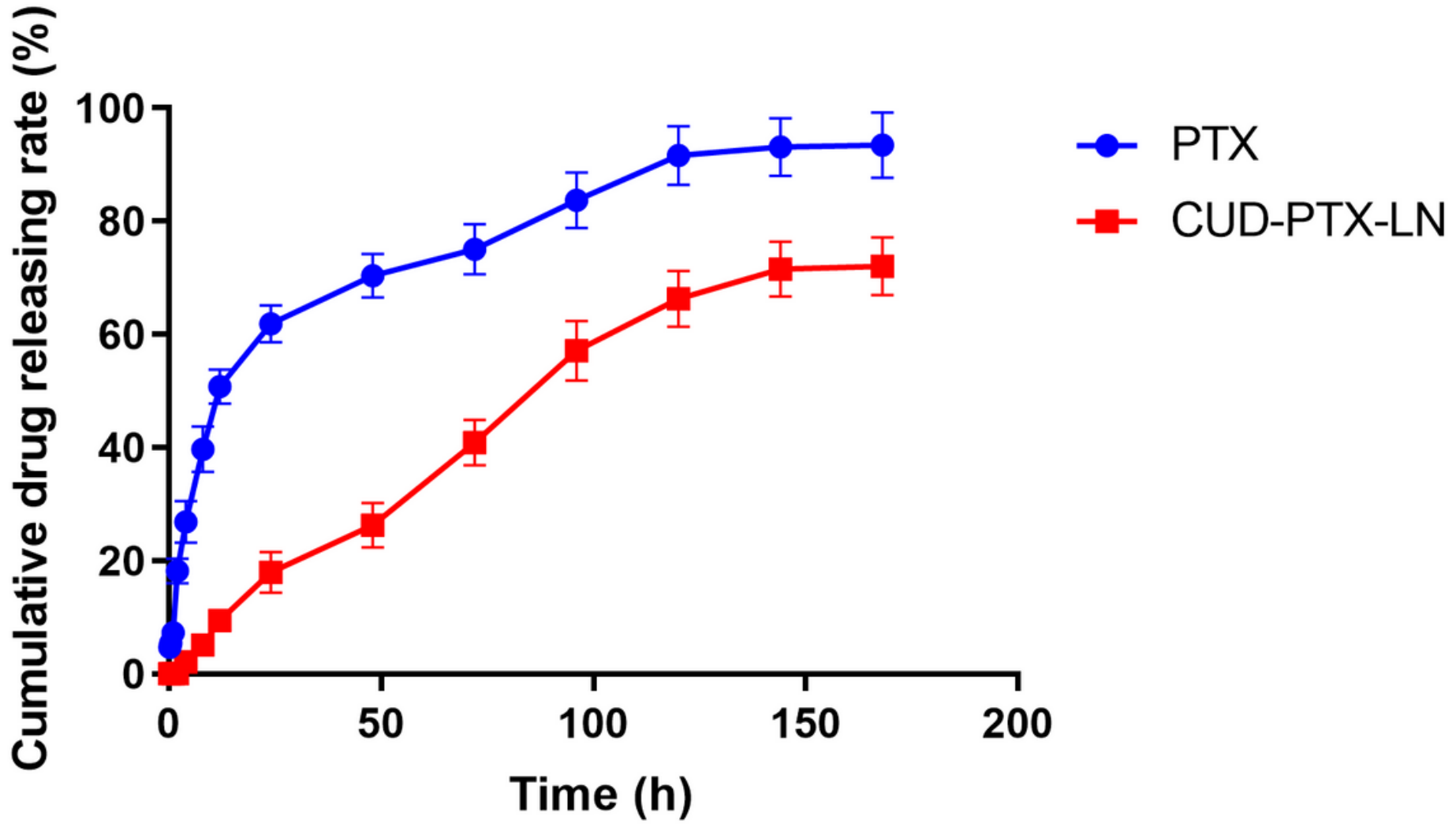

Figure 3 

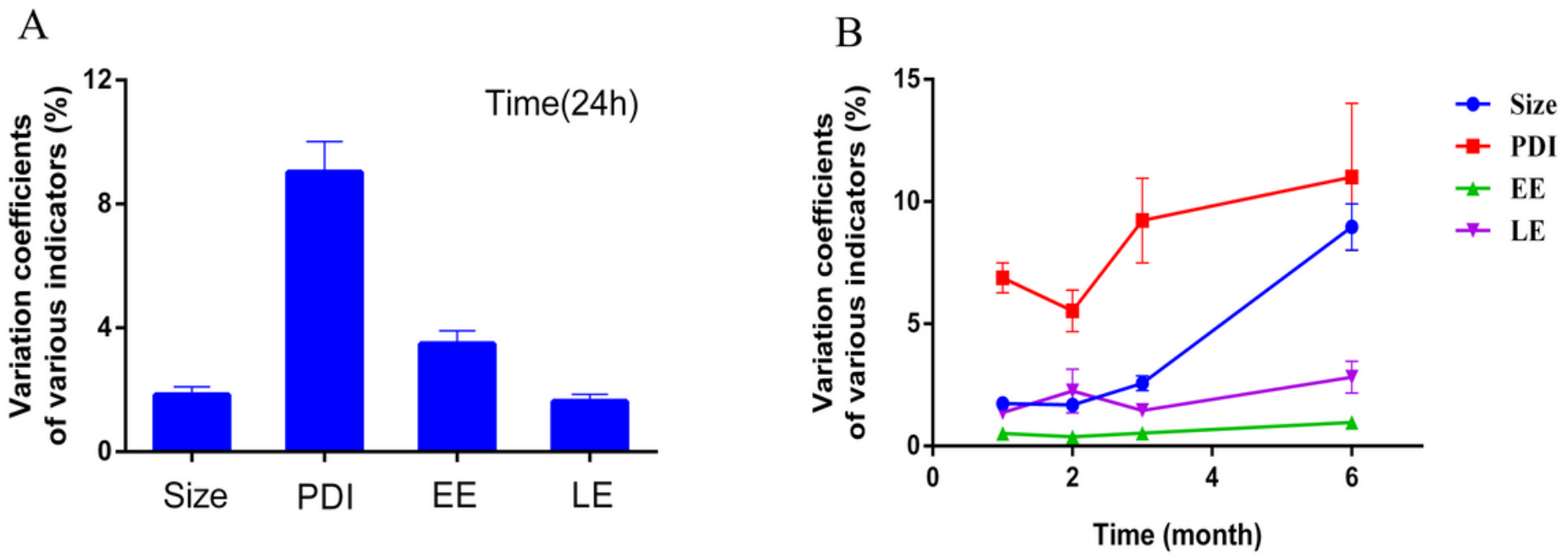

Figure 4

Caption not included with this version

A

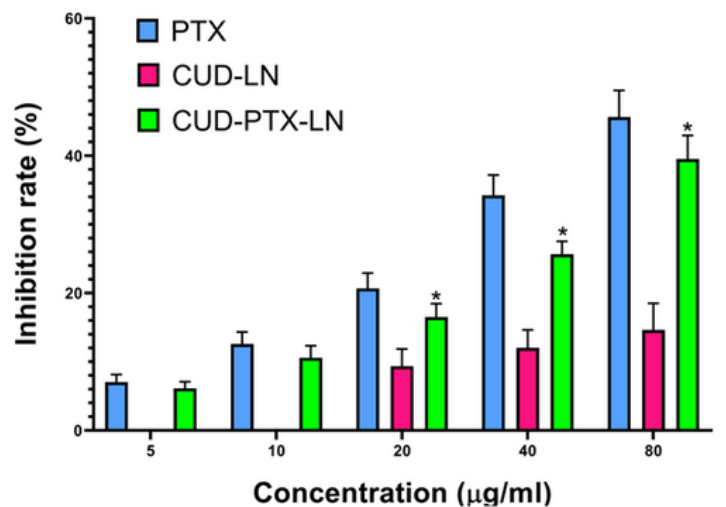

B

C
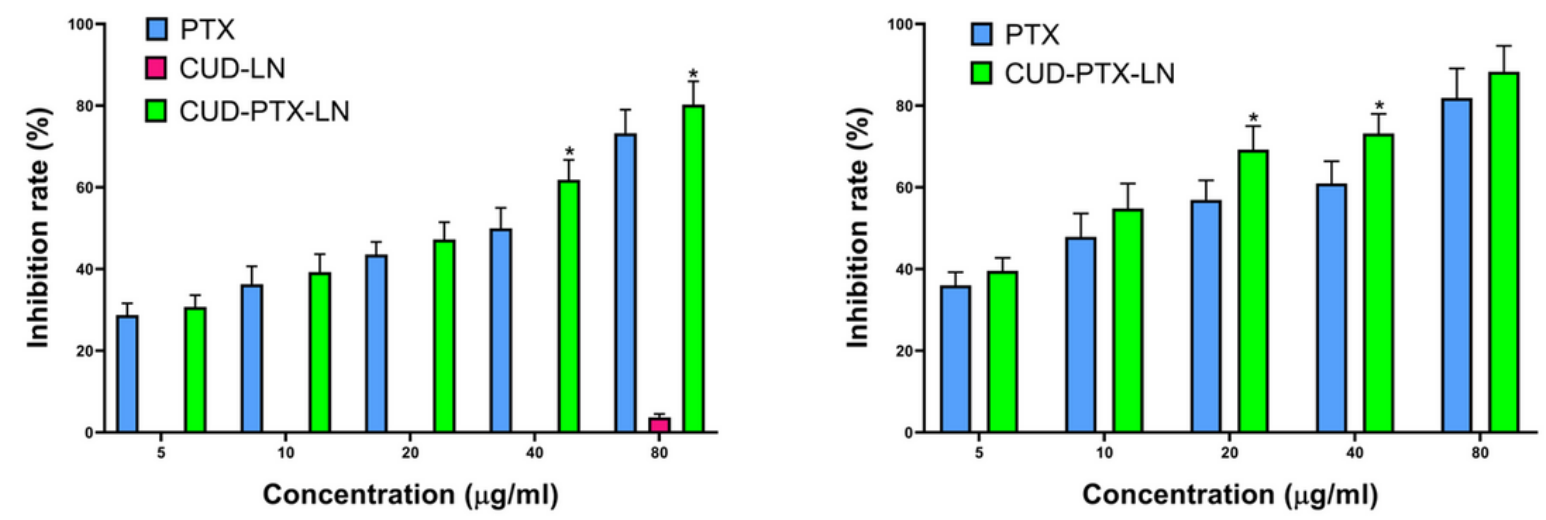

Figure 5

Caption not included with this version 
A

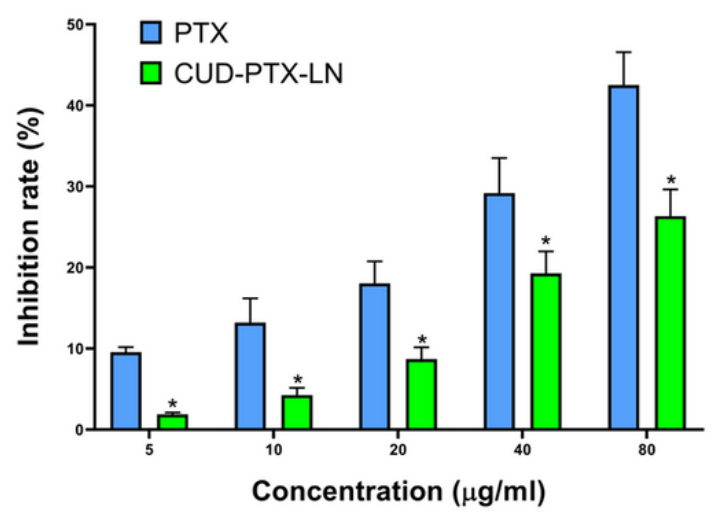

C

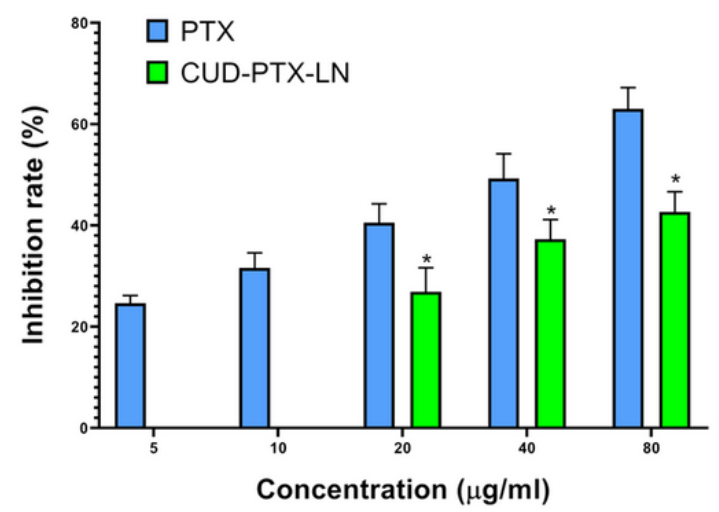

B

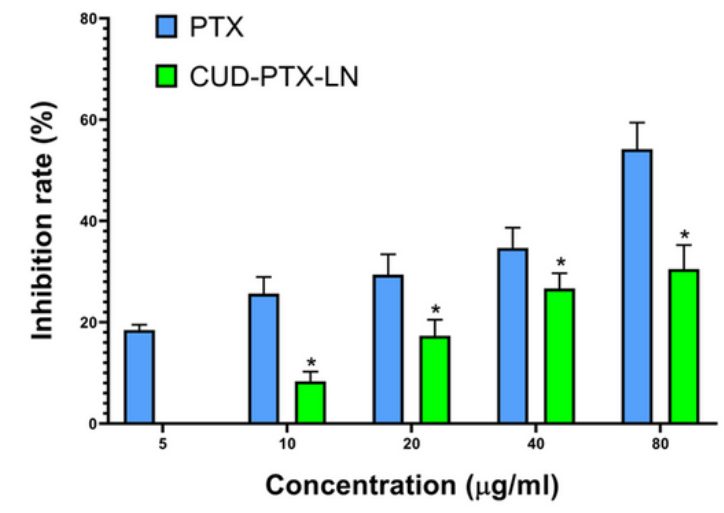

D

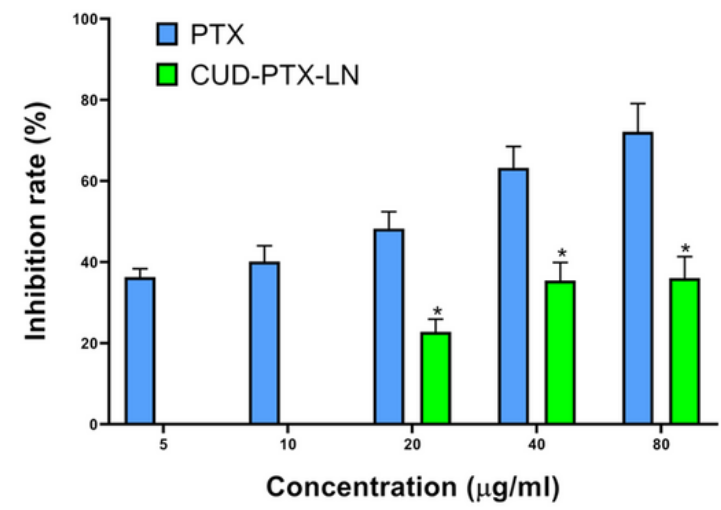

Figure 6

Caption not included with this version 
A

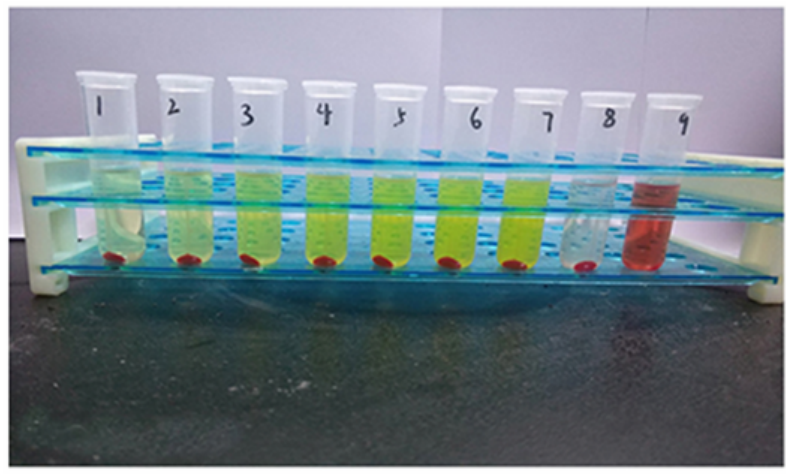

C

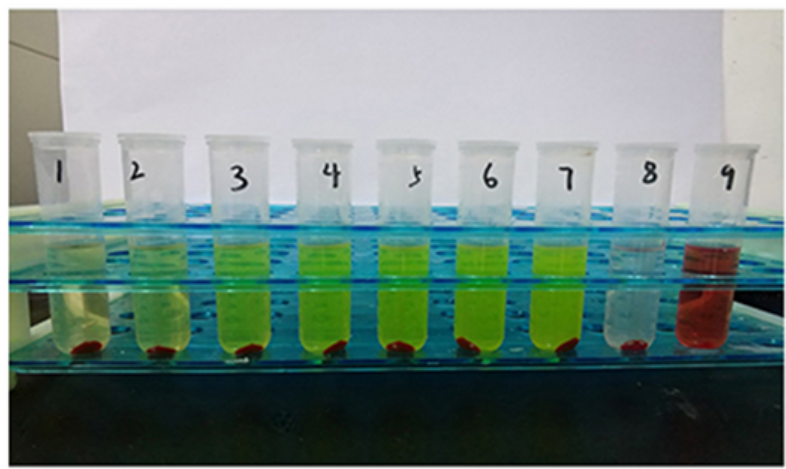

B

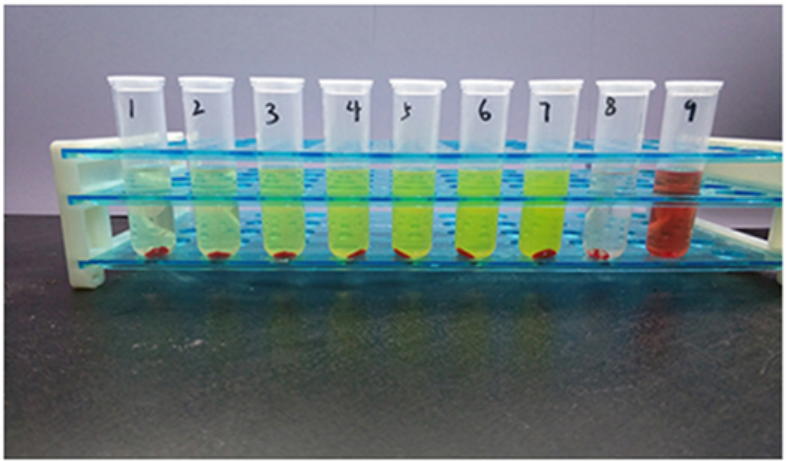

D

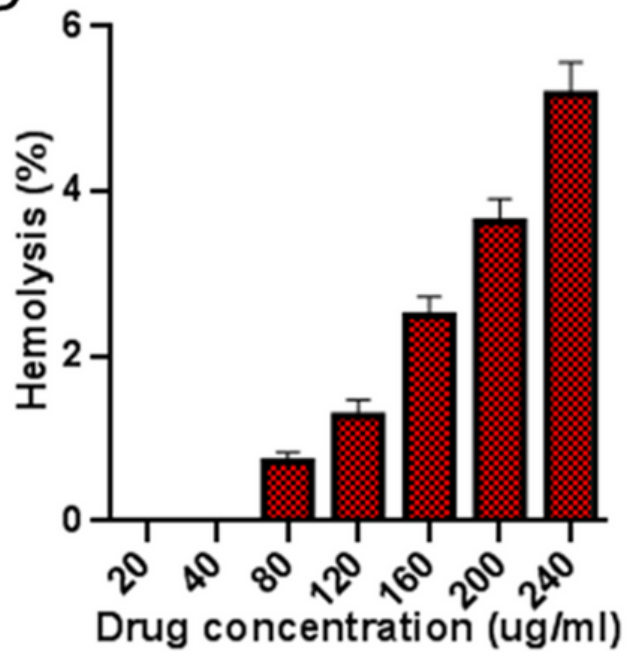

Figure 7

Caption not included with this version
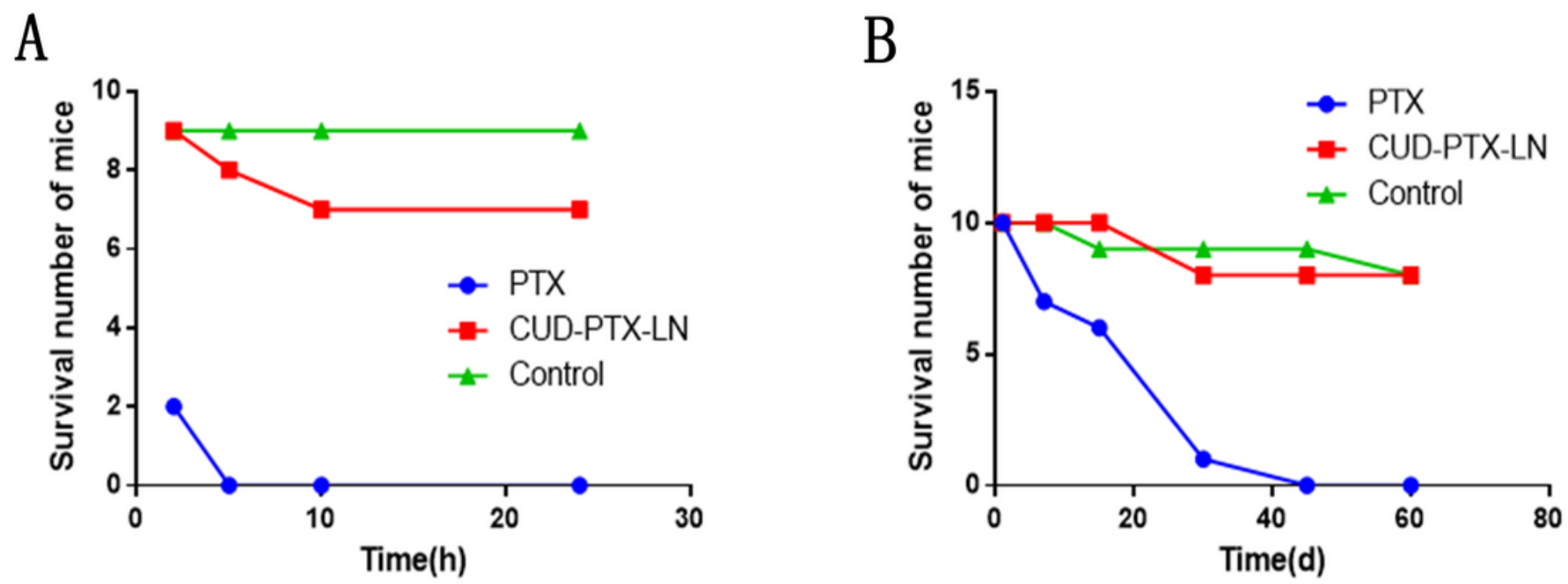

Figure 8

Caption not included with this version 
A
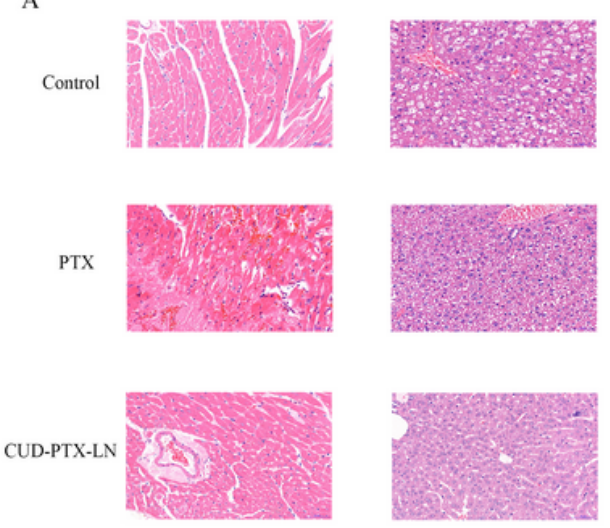

Heart

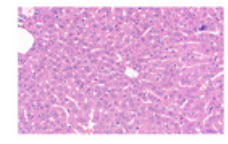

Liver
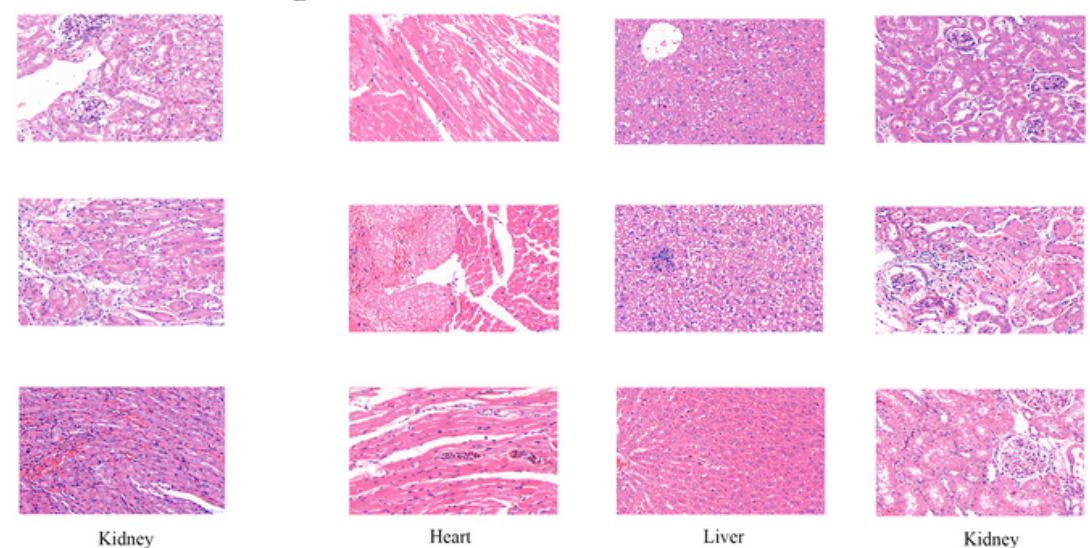

Liver

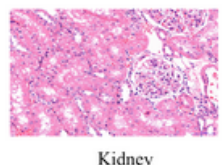

Figure 9

Caption not included with this version

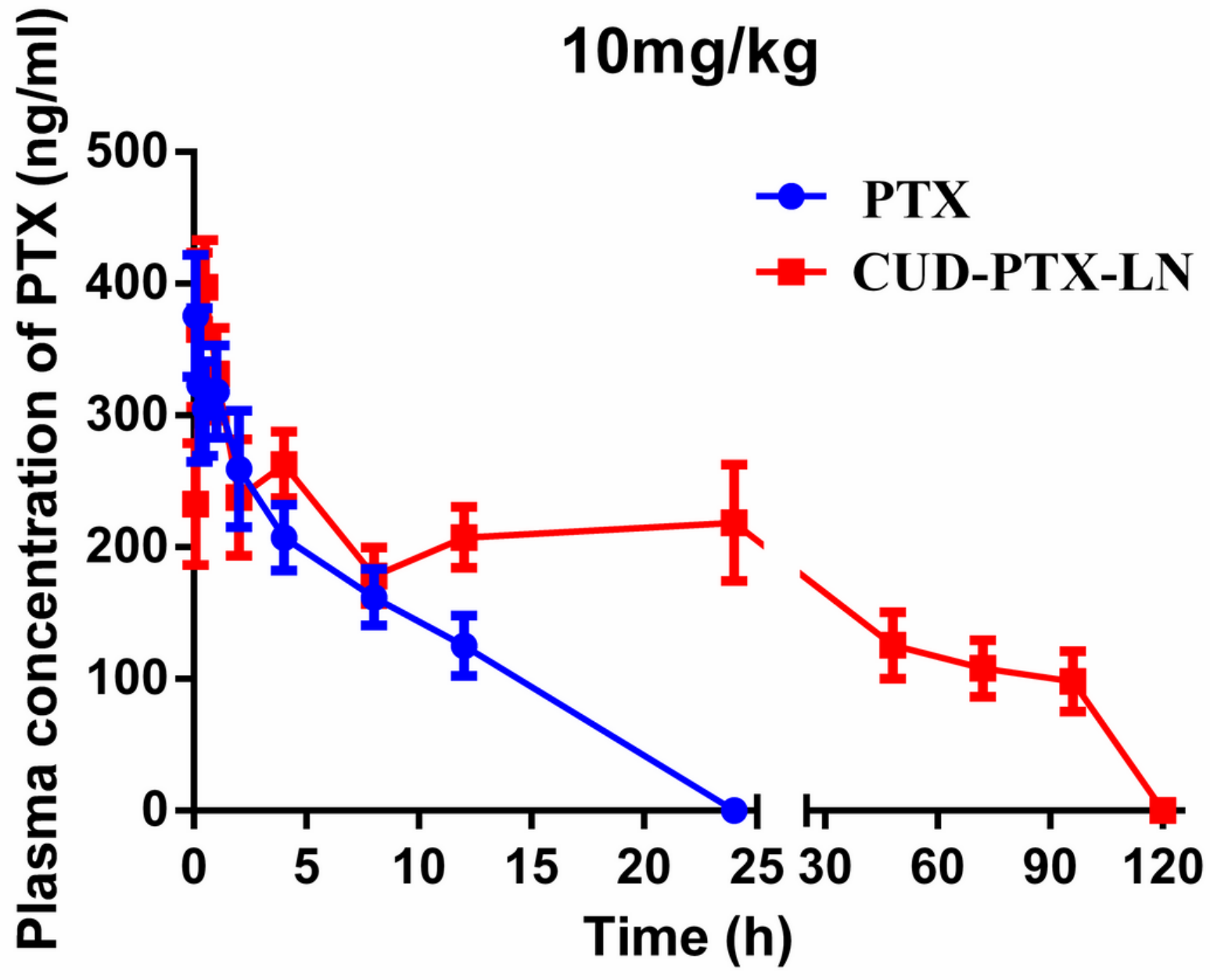

Figure 10

Caption not included with this version 
A

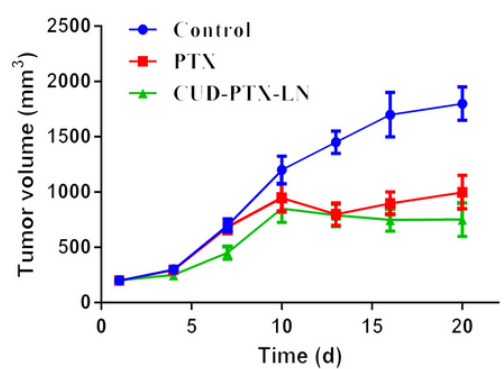

C

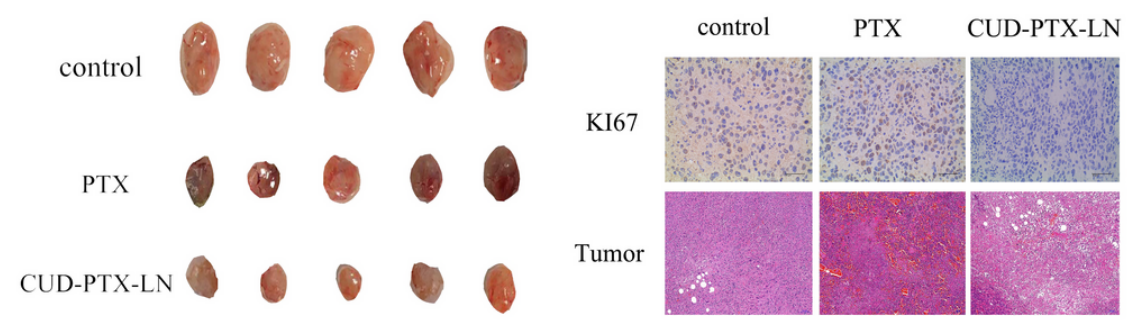

E

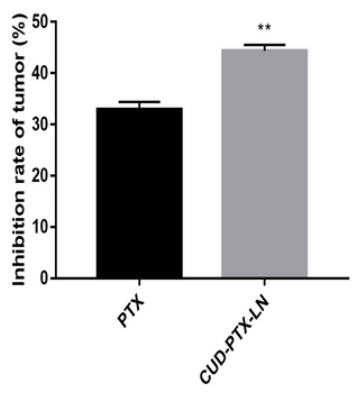

B

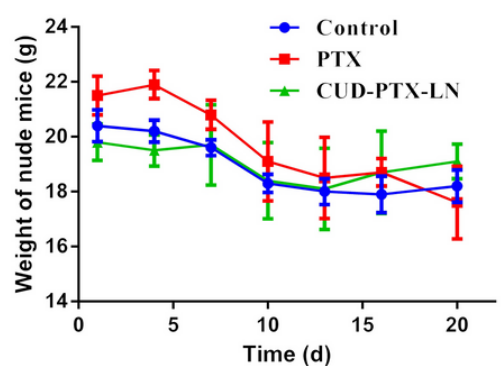

D

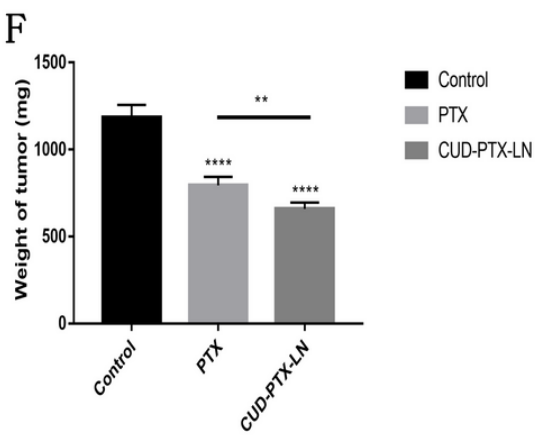

Figure 11

Caption not included with this version

\section{Supplementary Files}

This is a list of supplementary files associated with this preprint. Click to download.

- graphicalabstract.tif 